\title{
The study of phospholipid bilayer of cell membranes in leukocytes incubated with high concentrations of the food additive E407a
}

\author{
Anton Tkachenko ${ }^{1,2}$, Anatolii Onishchenko ${ }^{1.2}$, Alexander Roshal ${ }^{3}$, Yevgen Posokhov ${ }^{1,4}$ \\ ${ }^{I}$ Research Institute of Experimental and Clinical Medicine, Kharkiv National Medical University, Kharkiv, Ukraine \\ ${ }^{2}$ Department of Biochemistry, Kharkiv National Medical University, Kharkiv, Ukraine \\ ${ }^{3}$ Institute of Chemistry, V.N. Karazin Kharkiv National University, Kharkiv, Ukraine \\ ${ }^{4}$ Department of Organic Chemistry, Biochemistry and Microbiology, National Technical University "Kharkiv Polytechnic Institute”, Kharkiv, Ukraine
}

Received: 2021-01-30.

Accepted: 2021-03-08

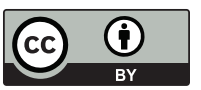

This work is licensed under a Creative Commons Attribution 4.0 International License

J Clin Med Kaz 2021; 18(2):49-52

Corresponding author:

Anton Tkachenko.

E-mail: antontkachenko555@gmail.com;

ORCID: http://www.orcid.org/0000-0002-1029-1636

\begin{abstract}
Aim: The aim of the study was to assess the impact of semi-refined carrageenan (E407a) on the hydrophobic region of phospholipid bilayer in cell membranes of leukocytes exposed to the solution with high concentrations of this food additive.
\end{abstract}

Material and methods: Fluorescent probe (2-phenylphenanthro[9,10-d]-1,3-oxazole) was used to investigate the influence of E407a on the state of lipid bilayer in leukocytes extracted from rats and treated with a 5\% solution of the food additive E407a during 4 hours.

Results: The shapes of the probe fluorescence spectra did not differ in leukocytes of rats treated with the 5\% solution of semi-refined carrageenan and white blood cells of control samples. Such findings suggest that exposure to the E407a solution causes no changes in the proton-donor ability of the media in the lipid membranes of leukocytes in the region where the probe locates.

Conclusion: Exposure of white blood cell suspensions to the semirefined carrageenan solution does not affect the membrane hydration of the hydrophobic region of leukocyte phospholipid bilayer.

Key words: fluorescence, phospholipids, polysaccharides, membrane fluidity, rats

\begin{abstract}
Introduction
Marine polysaccharides have been successfully used in food technology to improve the texture and appearance of food products as gelling agents, thickeners and stabilizers due to their hydrocolloid properties [1]. This group of compounds includes agar, alginates, and carrageenans. Carrageenans are the most abundant microalgae-derived polysaccharides in the food market. Thus, the safety and regulatory aspects of their consumption are of huge importance. At the moment, CGNs are being considered safe by the world-renowned food safety regulatory organizations such as the U.S. Food and Drug Administration (FDA), Joint Expert
\end{abstract}

Committee on Food Additives (JECFA), and European Food Safety Authority (EFSA) [2]. However, several studies have demonstrated that carrageenans are able to trigger intestinal inflammation as a result of their oral exposure [3-6]. This controversy of carrageenans has created grounds for re-evaluation of their safety in the food industry, and in 2018 the EFSA released a call for technical and toxicological data on carrageenan (E 407) for uses in foods for all population groups including infants below 16 weeks of age (EFSA-Q-number: EFSA-Q-2018-00771). According to this document, the corresponding data will be collected until the end of 2022. It is worth mentioning that the mechanisms by 
which carrageenans promote inflammation are still to be studied. However, there are some advances in elucidating such mechanisms. Both cell culture-based and animal-based studies have provided evidence that reactive oxygen species (ROS) are involved in carrageenan-mediated cytotoxicity [7-10]. Moreover, carrageenan-induced ROS production is believed to affect other pro-inflammatory intracellular signaling pathways, e.g. Bhattacharyya $S$ et al reported that in the carrageenantriggered signaling cascade a ROS downstream transcriptional factor in cells exposed to carrageenans is nuclear factor kappalight-chain-enhancer of activated B cells (NF-kB). In addition, ROS mediated IL-8 production in cells treated with carrageenans [10]. Besides, carrageenan has been reported to activate toll-like receptor 4 (TLR4)/Toll/IL-1R domain-containing adaptor-inducing IFN- $\beta$ (TRIF)/spleen tyrosine kinase (SYK)/ ROS signaling [11]. Thus, the role of ROS in carrageenaninduced toxicity is not limited to ROS-mediated damage to macromolecules. Carrageenan-triggered ROS overproduction mediates activation of pro-inflammatory signaling pathways that contribute to the development of carrageenan-induced inflammation. Given the role of immune cells in inflammation, the evaluation of the effects of carrageenans on leukocytes is extremely appreciated. It has been recently demonstrated that semi-refined carrageenan (E407a) promotes generation of ROS in leukocytes in laboratory animals upon oral administration and in vitro [7;9]. Since ROS are characterized by high chemical reactivity attributable to the presence of uncoupled electrons, they interact with fatty acids that are structural constituents of phospholipid bilayers in cell membranes [12]. Such ROSmediated lipid peroxidation affects structure of cell membranes changing lipid order, fluidity and viscosity of cell membranes, which may compromise the functions of cells. In its turn, fluidity of cell membranes determines cellular responses, including signaling and trafficking, and, thus, the maintenance of lipid order homeostasis in plasma membranes is of great importance [13]. Furthermore, leukocytes have extra function-associated features of proper plasma membrane organization. The particular lipid order of plasma membranes in killer T cells protects them, maintaining their ability to resist cytotoxic perforins, destroy target cells and survive during this process [14].

It has been earlier shown that incubation of leukocytes with semi-refined carrageenan promotes an enhancement in the lipid order of less hydrophobic area of phospholipid glycerol heads in cell membranes [15]. However, there is scarce data on the effects of carrageenans on deeper hydrophobic regions of cell membranes, in particular, areas of aliphatic acyl chains of fatty acids.

The aim of this study was to investigate the effects of direct exposure to E407a on the hydrophobic region of lipid membranes of leukocytes using a fluorescent probe.

\section{Material and methods Experimental procedures}

Blood was collected from 8 female WAG rats weighing 160-190 g into sterile vacutainer tubes containing dipotassium ethylenediaminetetraacetate (K2EDTA; IMPROVACUTER Evacuated EDTA K2 Spray Dried PET Tubes, Guangzhou, China). Thereafter, two aliquots of $500 \mu \mathrm{l}$ were transferred from each vacutainer tube to polysterene tubes for further incubation. Experimental samples were incubated with $100 \mu$ of semi-refined carrageenan solution in phosphate buffered saline (PBS, $\mathrm{pH} 7.4$ ) (Becton Dickinson, USA) reaching the final concentration of dry matter equal to $5 \%$ during $4 \mathrm{~h}$. Control samples were incubated with PBS in the same conditions.
The European Convention for the Protection of Vertebrate Animals used for Experimental and other Scientific Purposes (EST 123), Directive 2010/63/EU for the Protection of Animals Used for Scientific Purposes, and Recommendation 2007/526/ EC regarding guidelines for the accommodation and care of animals used for experimental and other scientific purposes were followed. The study was approved by the Commission on Ethics and Bioethics (Kharkiv National Medical University, Kharkiv, Ukraine; minutes \#5 dated September 17, 2019).

\section{Fluorescent probe PH1}

The cells were fluorescently labeled in accordance with the following procedure: an aliquot of the probe stock solution in acetonitrile was added to the leukocyte suspension to achieve a final probe concentration of $\sim 5 \times 10^{-6} \mathrm{~mol} / \mathrm{L}$. Lipid-to-probe molar ratio was $\sim 200: 1$. Before fluorescence measurements, the cell suspensions were incubated with the probe at room temperature for 1 hour.

The fluorescence spectra were measured on a fluorometer Hitachi F850 (Tokyo, Japan) in the range of 350-630 nm, with an increment of $2 \mathrm{~nm}$. The excitation wavelength was $330 \mathrm{~nm}$. The excitation and emission slits were $5 \mathrm{~nm}$.

Fluorescent probe PH1 (2-phenyl-phenanthro[9,10-d]-1,3oxazole) was used since the shape of its fluorescence spectrum depends upon the proton-donor ability of the microenvironment $[16 ; 17]$. The ratio of the intensities of the most short-wavelength fluorescence band and the most long-wavelength fluorescence band $\left(\mathrm{I}_{S} / \mathrm{I}_{\mathrm{L}}\right)$ of probe $\mathrm{PH} 1$ was used as a parameter for estimation of the changes in the proton-donor ability of the probe microenvironment: e.g., with growth of the proton-donor ability of the media, the $\mathrm{I}_{S} / \mathrm{I}_{L}$ ratio decreases $[16 ; 17]$.

The expected location of probe PH1 in lipid membranes: the area of hydrocarbon chains of phospholipids of the lipid bilayer and the centre of the lipid bilayer [16;17]. Its location is demonstrated in Figure 1.

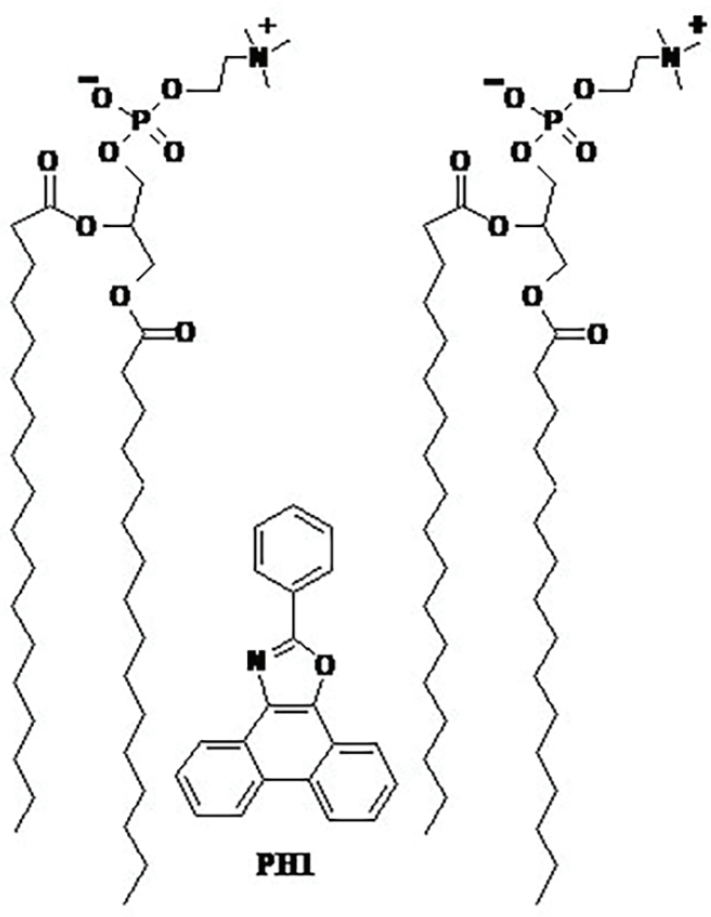

Figure 1. The figure demonstrates localization and orientation of fluorescent probe $\mathrm{PH} 1$ in phospholipid membranes. Two molecules of phosphatidylcholine from the outer leaflet are shown to denote the localization of the probe (Adapted from Ref [16]). 
Since water has the proton-donor ability and penetrates into lipid bilayers, fitting packing defects between fatty acyl chains [18;19], thus constituting an interchain hydration, the probe can be used to detect the changes in the hydration of lipid membranes [16;17].

Given that the changes in membrane hydration, in their turn, are linked with the changes of the membrane lipid order [20-22], the probe can indicate such modifications.

\section{Statistics}

The non-parametric Mann-Whitney U test was chosen to compare data in two independent groups of samples. The median (Me) and interquartile range (IQR; 25\%-75\%) were presented as numerical parameters to compare differences in the fluorescence of fluorescent probe PH1. The differences were statistically significant if $\mathrm{p}$ values were below 0.05. Graph Pad Prism 5.0 was used to process data.

\section{Results}

The shape of the spectra of fluorescent probe PH1 in leukocyte cell membranes did not differ between leukocytes treated with high concentrations of the common food additive E407a and the corresponding control samples containing leukocytes isolated from intact animals (Figure 2).

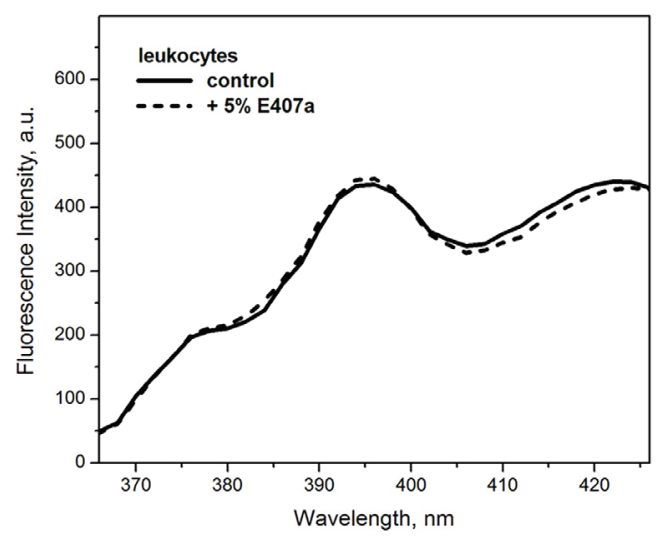

Figure 2. Representative fluorescence spectra of probe $\mathrm{PH}$ in leukocyte suspensions: (a) control samples containing leukocytes obtained from the intact rats (solid line), (b) leukocytes treated with the 5\% E407a solution during $4 \mathrm{~h}$ (dashed line).

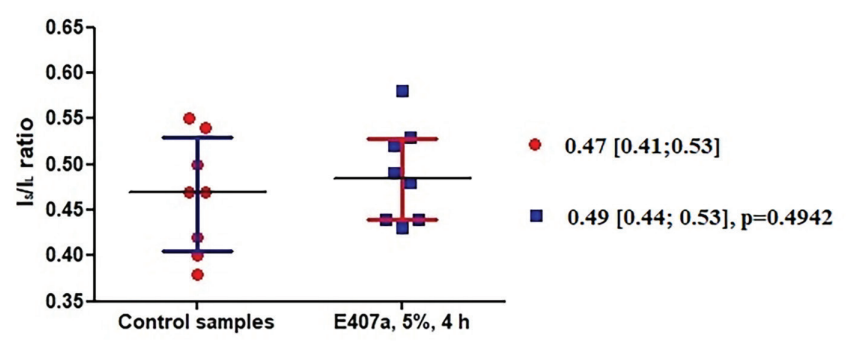

Figure 3. The ratio of the intensities of the most short-wavelength fluorescence band and the most long-wavelength fluorescence band (IS/IL) of probe PHI in leukocyte suspensions treated and untreated with the food additive E407a

Two parameters that characterize how probes are embedded in cell membranes were analyzed. Comparison of numerical values of $I_{S} / I_{L}$ ratios demonstrated no significant difference (Figure 3) indicating that exposure to the solution of E407a did not affect the proton-donor ability in WBC cell membranes of rats in the membrane area, where probe $\mathrm{PH} 1$ locates, e.g. in the hydrophobic region of the lipid bilayer. The values were 0.49
$[0.44 ; 0.53]$ and $0.47[0.41 ; 0.53]$ in samples treated with E407a and PBS, respectively. No statistical significance was observed $(\mathrm{p}=0.49)$.

Incubation of leukocytes with semi-refined carrageenan has no effect on the values of the intensity of fluorescence maximum (I395). This index was 446 [437; 445] a.u. in the experimental samples, while in the control group the values were 436 [422; 442] a.u. Data are available in Figure 4. The difference was statistically insignificant $(\mathrm{p}=0.07)$.

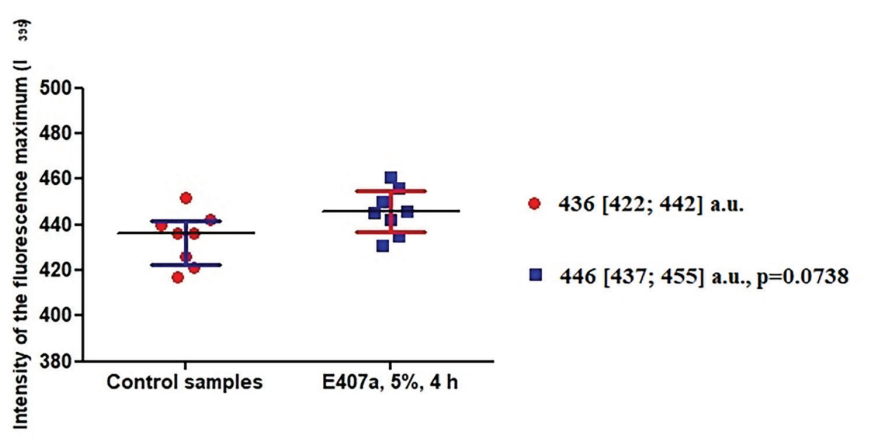

Figure 4. The intensity of the fluorescence maximum of the probe $\mathrm{PH} 1$ in cell membranes of intact leukocytes and white blood cells incubated with E407a (5\%)

\section{Discussion}

Our experimental findings, i.e. the absence of the changes in the shape of the fluorescence spectra, indicate that no changes in the membrane hydration are detected in the area of hydrocarbon chains of phospholipids and the centre of the lipid bilayer of the cell membranes in leukocytes after exposure to the $5 \%$ solution of the food additive E407a during 4 hours. The discussed lack of the changes in the membrane hydration points to the absence of the changes in the lipid order of the hydrophobic region of the leukocyte membrane.

These experimental data are consistent with our earlier results that have demonstrated the impact of the incubation of WBCs with E407a on more polar regions of leukocyte cell membranes [15]. The decrease in the polarity and the protondonor ability of the microenvironment of fluorescent probes O1O (2-(2'-hydroxy-phenyl)-5-phenyl-1,3-oxazole) and O6O (2-(2'-hydroxy-phenyl)-5-(4'-biphenyl)-1,3-oxazole) were indicative of the reduction in hydration in the area of glycerol backbones and carbonyl groups of phospholipids and, thus, suggested the enhanced membrane lipid order.

Given the structure of phospholipid-based cell membranes with hydrophilic heads outward, it is clear that free radicals initially come in contact with the head groups of phospholipids. Thereafter, the ROS-mediated oxidation of phospholipid heads facilitates the subsequent oxidation of hydrophobic aliphatic tails. Yusupov M et al state that ROS oxidation of the tails is preceded by oxidation of the phospholipid heads [23]. It is interesting to note that hydroxyl and hydroperoxyl radicals, as well as hydrogen peroxide tend to locate near phospholipid heads, whereas molecular oxygen penetrates to the interior of cell membranes [24]. Taken together, our data suggest that carrageenan causes changes to the cell membranes similar to those observed due to their lipid peroxidation. However, unexpectedly, this process affects preferably the hydrophilic heads of phospholipids without affecting the hydrophobic fatty acid tails. It can be hypothesized that this can be attributable to the carrageenan-induced generation of those ROS that preferentially interact with heads. However, further research at the atomic scale 
is required to reveal how carrageenan-associated ROS promote lipid peroxidation.

\section{Conclusion}

Direct exposure of leukocytes to semi-refined carrageenan does not modify the hydration in hydrophobic regions of phopholipid bilayer.
Disclosures: There is no conflict of interest for all authors.

Acknowledgements:

The study was performed as a fragment of the research entitled "Biochemical Mechanisms for the Induction of Intestinal Inflammation and the Ways of its Correction (Kharkiv National Medical University, Kharkiv, Ukraine; state registration number 0120U102645).

Funding: The research was not funded in any way.

\section{References}

1. Ruocco N, Costantini S, Guariniello S, Costantini M. Polysaccharides from the Marine Environment with Pharmacological, Cosmeceutical and Nutraceutical Potential. Molecules. 2016; 21(5):551. doi: 10.3390/molecules21050551.

2. Weiner ML, McKim JM. Comment on "revisiting the carrageenan controversy: do we really understand the digestive fate and safety of carrageenan in our foods?” by S. David, C. S. Levi, L. Fahoum, Y. Ungar, E. G. Meyron-Holtz, A. Shpigelman and U. Lesmes. Food Funct. 2018; 9:1344-1352. Food Funct. 2019; 10(3):1760-2. https://doi.org/10.1039/c8fo01282b.

3. Tkachenko A, Marakushyn D, Kalashnyk I, Korniyenko Y, Onishchenko A, Gorbach T, et al. A study of enterocyte membranes during activation of apoptotic processes in chronic carrageenan-induced gastroenterocolitis. Med Glas (Zenica). 2018; 15(2):87-92. doi: 10.17392/946-18

4. Gubina-Vakyulyk GI, Gorbach TV, Tkachenko AS, Tkachenko MO. Damage and regeneration of small intestinal enterocytes under the influence of carrageenan induces chronic enteritis. Comparative Clinical Pathology. 2015; 24(6):1473-1477. https://doi.org/10.1007/ s00580-015-2102-3

5. Necas J, Bartosikova L. Carrageenan: a review. Veterinarni Medicina 2013; 58:187-205.

6. Tobacman JK. Review of harmful gastrointestinal effects of carrageenan in animal experiments. Environ Health Perspect. 2001; 109(10):983-994. doi:10.1289/ehp.01109983

7. Tkachenko AS, Kot YG, Kapustnik VA, Myasoedov VV, Makieieva NI, Chumachenko TO, Onishchenko AI, Lukyanova YM, Nakonechna OA. Semi-refined carrageenan promotes reactive oxygen species (ROS) generation in leukocytes of rats upon oral exposure but not in vitro. Wien Med Wochenschr. 2020. https://doi.org/10.1007/s10354-020-00786-7

8. Barth CR, Funchal GA, Luft C, de Oliveira JR, Porto BN, Donadio MV. Carrageenan-induced inflammation promotes ROS generation and neutrophil extracellular trap formation in a mouse model of peritonitis. Eur J Immunol. 2016; 46(4):964-70. doi: 10.1002/ eji.201545520.

9. Sokolova EV, Karetin Y, Davydova VN, Byankina AO, Kalitnik AA, Bogdanovich LN, Yermak IM. Carrageenans effect on neutrophils alone and in combination with LPS in vitro. J Biomed Mater Res A. 2016; 104(7):1603-9. doi: 10.1002/jbm.a.35693.

10. Bhattacharyya S, Dudeja PK, Tobacman JK. Carrageenan-induced NFkappaB activation depends on distinct pathways mediated by reactive oxygen species and Hsp27 or by Bcl10. Biochim Biophys Acta. 2008; 1780(7-8):973-982. doi:10.1016/j.bbagen.2008.03.019

11. Lopes AH, Silva RL, Fonseca MD, et al. Molecular basis of carrageenan-induced cytokines production in macrophages. Cell Commun Signal. 2020; 18(1):141. doi: 10.1186/s12964-020-00621-x.

12. Su LJ, Zhang JH, Gomez H, Murugan R, Hong X, Xu D, Jiang F, Peng ZY. Reactive Oxygen Species-Induced Lipid Peroxidation in Apoptosis, Autophagy, and Ferroptosis. Oxid Med Cell Longev. 2019; 2019:5080843. doi: 10.1155/2019/5080843.

13. Poggi P, Mirabella R, Neri S, Assirelli E, Dolzani P, Mariani E, Calder PC, Chatgilialoglu A. Membrane fatty acid heterogeneity of leukocyte classes is altered during in vitro cultivation but can be restored with ad-hoc lipid supplementation. Lipids Health Dis. 2015; 14:165. doi: 10.1186/s12944-015-0166-3.

14. Rudd-Schmidt JA, Hodel AW, Noori T, Lopez JA, Cho HJ, Verschoor S, Ciccone A, Trapani JA, Hoogenboom BW, Voskoboinik I. Lipid order and charge protect killer T cells from accidental death. Nat Commun. 2019; 10(1):5396. doi: 10.1038/s41467-019-13385-X.

15. Tkachenko A, Onishchenko A, Roshal A, Nakonechna O, Chumachenko T, Posokhov Y. Effects of semi-refined carrageenan (food additive E407a) on cell membranes of leukocytes assessed in vivo and in vitro. Med Glas (Zenica). 2021;18(1). doi: 10.17392/1213-21.

16. Posokhov YO, Kyrychenko A, Korniyenko Y. Derivatives of 2,5-diaryl-1,3-oxazole and 2,5-diaryl-1,3,4-oxadiazole as environmentsensitive fluorescent probes for studies of biological membranes. Reviews in Fluorescence 2017 (editor C.D. Geddes). Springer Nature Switzerland AG. 2018; 199-230.

17. Posokhov Y. Fluorescent probes sensitive to changes in the cholesterol-to-phospholipids molar ratio in human platelet membranes during atherosclerosis. Methods and Applications in Fluorescence. 2016; 4:034013.

18. Ho C, Stubbs CD. Hydration at the membrane protein-lipid interface. Biophysical Journal. 1992; 63:897-902.

19. Disalvo EA, Bouchet AM, Frias MA. Connected and isolated $\mathrm{CH} 2$ populations in acyl chains and its relation to pockets of confined water in lipid membranes as observed by FTIR spectrometry. Biochimica et Biophysica Acta. 2013; 1828:1683-1689.

20. Ho C, Slater SJ, Stubbs CD. Hydration and order in lipid bilayers. Biochemistry 1995;34:6188- 6195.

21. Binder H, Gawrisch K. Effect of unsaturated lipid chains on dimensions, molecular order and hydration of membranes. J. Phys. Chem. B. 2001; 105:12378- 12390 .

22. Noethig-Laslo V, Šentjurc M. Transmembrane polarity profile of lipid membranes. Advances in Planar Lipid Bilayers and Liposomes. Academic Press, Elsevier. 2006; 365-415.

23. Yusupov M, Wende K, Kupsch S, Neyts EC, Reuter S, Bogaerts A. Effect of head group and lipid tail oxidation in the cell membrane revealed through integrated simulations and experiments. Sci Rep. 2017; 7(1):5761. doi: 10.1038/s41598-017-06412-8.

24. Cordeiro RM. Reactive oxygen species at phospholipid bilayers: distribution, mobility and permeation. Biochim Biophys Acta. 2014; 1838(1 Pt B):438-44. doi: 10.1016/j.bbamem.2013.09.016. 